\title{
Effects of density imbalance on the BCS-BEC crossover in semiconductor electron-hole bilayers
}

\author{
P. Pieri, ${ }^{1}$ D. Neilson, ${ }^{1,2}$ and G.C. Strinati ${ }^{1}$ \\ ${ }^{1}$ Dipartimento di Fisica, Università di Camerino, I-62032 Camerino, Italy \\ ${ }^{2}$ School of Physics, University of New South Wales, Sydney 2052, Australia
}

(Dated: March 23, 2022)

\begin{abstract}
We study the occurrence of excitonic superfluidity in electron-hole bilayers at zero temperature. We not only identify the crossover in the phase diagram from the BCS limit of overlapping pairs to the BEC limit of non-overlapping tightly-bound pairs but also, by varying the electron and hole densities independently, we can analyze a number of phases that occur mainly in the crossover region. With different electron and hole effective masses, the phase diagram is asymmetric with respect to excess electron or hole densities. We propose as the criterion for the onset of superfluidity, the jump of the electron and hole chemical potentials when their densities cross.
\end{abstract}

PACS numbers: 03.75.Hh,03.75.Ss,73.63.Hs

There is a growing interest in the physics of the BCSBEC crossover, owing to the recent experimental advances with trapped Fermi atoms. With the use of FanoFeshbach resonances, this crossover has been observed with fermionic ${ }^{6} \mathrm{Li}$ and ${ }^{40} \mathrm{~K}$ atoms $[1]$ which become composite bosons in the BEC limit. Although trapped atoms represent an ideal testing ground for a fundamental understanding of the BCS-BEC crossover, technological applications exploiting the occurrence of condensates will most probably rely on semiconductor systems. In these systems, excitons made up of electrons and holes play the role of composite bosons.

Excitonic systems were, in fact, the first to be considered for the BCS-BEC crossover. In bulk materials, original work on exciton condensation was done by Keldysh and co-workers [2]. Extension of this to the BCS-BEC crossover was proposed by Nozières and Comte [3]. However, in bulk materials fast electron-hole recombination hinders the detection of BEC. Proposals have accordingly been made to condense excitons with spatially separated electrons and holes [4, 5]. Particularly promising are bilayer quantum-well systems separated by a distance $d$, with conduction-band electrons in one well and valenceband holes in the adjacent well [6, 7, 8, 9]. Recently, developments for the detection of excitonic BEC in coupled quantum wells has been reported 10, 11, 12. . Technological advances with electron-hole bilayers [13] make it now possible to contact separately the layers of electrons and holes in GaAs, separated by an AlGaAs barrier of thickness $d$ as small as $15 \mathrm{~nm}$.

In this paper, we consider the BCS-BEC crossover in electron-hole bilayers when the densities of the electrons and holes are varied independently of each other. The effect of the density imbalance resembles that of a magnetic field in a superconductor (disregarding orbital effects) first considered by Sarma [14]. This analogy was also noted in Ref. [15], where the influence of an isospin asymmetry in nuclear matter was considered for the BCS-BEC crossover. The specific system we shall consider as an ex- ample is GaAs-AlGaAs.

Recent experiments with population imbalance in ultracold trapped Fermi atoms [16] have stimulated a considerable amount of theoretical work on two-component Fermi systems with density imbalance [17, 18]. Emphasis has been placed on the possible occurrence of exotic phases in addition to the ordinary BCS pairing [17, 19, 20]. However, in these systems the presence of a trap and their charge neutrality inhibit the occurrence of the exotic phases, and so far only phase separation between a superfluid core with equally matched populations and an outer normal phase has been detected.

Electron-hole bilayers may offer a better opportunity of observing such exotic phases because the Coulomb repulsion within each layer acts to suppress phase separation 21]. In addition, we find that the different electron and hole effective masses in GaAs, $m_{\mathrm{e}}$ and $m_{\mathrm{h}}$, and also the non-local nature of the electron-hole attraction both favor the occurrence of exotic phases. These include the Sarma phase with one or two Fermi surfaces and finite population imbalance, and the Fulde-FerrelLarkin-Ovchinnikov (FFLO) phase 22], as well as the ordinary BCS pairing with equal populations. The relative extension of these phases is quite asymmetric between an imbalance with more holes and an imbalance with more electrons. We also find that in the superfluid phase the separate electron and hole chemical potentials display a jump when reversing the population imbalance from more electrons to more holes, while in the normal phase no jump occurs. Detection of this jump could thus serve to identify superfluid character in a system.

The electron-hole bilayer system is described by the grand-canonical Hamiltonian:

$$
\begin{aligned}
& K=\sum_{\mathbf{k}, \sigma} \xi_{\mathbf{k} \sigma} c_{\mathbf{k} \sigma}^{\dagger} c_{\mathbf{k} \sigma}+\frac{1}{2 \Omega} \sum_{\mathbf{k}, \mathbf{k}^{\prime}, \mathbf{q}, \sigma, \sigma^{\prime}} V_{\mathbf{k}-\mathbf{k}^{\prime}}^{\sigma \sigma^{\prime}} \\
& \times c_{\mathbf{k}+\frac{\mathbf{q}}{2} \sigma}^{\dagger} c_{-\mathbf{k}+\frac{\mathbf{q}}{2} \sigma^{\prime}}^{\dagger} c_{-\mathbf{k}^{\prime}+\frac{\mathbf{q}}{2} \sigma^{\prime}} c_{\mathbf{k}^{\prime}+\frac{\mathbf{q}}{2} \sigma} .
\end{aligned}
$$

Here, $\mathbf{k}, \mathbf{k}^{\prime}$, and $\mathbf{q}$ are two-dimensional wave vectors in 
the layers, $\Omega$ is the quantization volume (surface area), $c_{\mathbf{k} \sigma}^{\dagger}\left(c_{\mathbf{k} \sigma}\right)$ are the creation (destruction) operators for electrons (e) and holes (h) distinguished by $\sigma=(\mathrm{e}, \mathrm{h})$, the $\xi_{\mathbf{k} \sigma}=\epsilon_{\mathbf{k} \sigma}-\mu_{\sigma}$ are the band dispersions with chemical potentials $\mu_{\sigma}$ for electrons and holes, $\epsilon_{\mathbf{k e}}=\mathbf{k}^{2} /\left(2 m_{\mathrm{e}}\right)+E_{\mathrm{g}}$ and $\epsilon_{\mathrm{h}}(\mathbf{k})=\mathbf{k}^{2} /\left(2 m_{\mathrm{h}}\right)$. The semiconductor band gap $E_{\mathrm{g}}$ can be reabsorbed in the electron chemical potential. Explicit spin quantum numbers are omitted.

We have carried out our calculations in the zerotemperature limit, where a mean-field description of the BCS-BEC crossover is appropriate even in two dimensions [23]. We use an unscreened electron-hole attractive potential as in Ref. 7], $V_{\mathrm{k}}^{\mathrm{eh}}=-2 \pi e^{2} \exp (-k d) /(k \varepsilon)$, where $k=|\mathbf{k}|, e$ is the electron charge, and $\varepsilon$ the background dielectric constant. The gap in the superfluid phase makes screening less effective, so introducing screening should only cause small quantitative changes.

In the present calculation we neglect the intra-layer Coulomb repulsions $V^{\mathrm{ee}}$ and $V^{\mathrm{hh}}$. In the absence of density imbalance, $V^{\mathrm{ee}}$ and $V^{\mathrm{hh}}$ can be readily included in a mean-field treatment 7], but when the densities are imbalanced, including $V^{\text {ee }}$ and $V^{\mathrm{hh}}$ in the mean field requires a detailed knowledge of how overall charge neutrality is attained. This is in order to avoid divergence of the Hartree term. This depends on the specific engineering configuration of the device, and therefore in the interests of generality we drop $V^{\mathrm{ee}}$ and $V^{\mathrm{hh}}$ here. When the densities are equal we can compare with Ref. [7], and we have verified that omitting $V^{\mathrm{ee}}$ and $V^{\mathrm{hh}}$ reduces the size of the gap by an amount no larger than $30 \%$. As a separate issue, and as we have already noted, intra-layer repulsion should stabilize the system against phase separation. For this reason, we exclude the possibility of phase separation from our discussion of the phase diagram.

The relevant mean-field equations to be solved for the variables $\mu_{\mathrm{e}}, \mu_{\mathrm{h}}$, and the (s-wave) gap function $\Delta_{\mathbf{k}}$ are:

$$
\begin{aligned}
\Delta_{\mathbf{k}} & =-\frac{1}{\Omega} \sum_{\mathbf{k}^{\prime}} V_{\mathbf{k}-\mathbf{k}^{\prime}}^{\mathrm{eh}} \frac{\Delta_{\mathbf{k}^{\prime}}}{2 E_{\mathbf{k}^{\prime}}}\left[1-f\left(E_{\mathbf{k}^{\prime}}^{+}\right)-f\left(E_{\mathbf{k}^{\prime}}^{-}\right)\right], \\
n_{\mathrm{e}} & =\frac{1}{\Omega} \sum_{\mathbf{k}}\left[u_{\mathbf{k}}^{2} f\left(E_{\mathbf{k}}^{+}\right)+v_{\mathbf{k}}^{2}\left(1-f\left(E_{\mathbf{k}}^{-}\right)\right)\right], \\
n_{\mathrm{h}} & =\frac{1}{\Omega} \sum_{\mathbf{k}}\left[u_{\mathbf{k}}^{2} f\left(E_{\mathbf{k}}^{-}\right)+v_{\mathbf{k}}^{2}\left(1-f\left(E_{\mathbf{k}}^{+}\right)\right)\right],
\end{aligned}
$$

where $f(E)=\Theta(-E)$ is the Fermi function at zero temperature, $E_{\mathbf{k}}=\sqrt{\xi_{\mathbf{k}}^{2}+\Delta_{\mathbf{k}}^{2}}$ with $\xi_{\mathbf{k}}=\left(\xi_{\mathbf{k}}^{\mathrm{e}}+\xi_{\mathbf{k}}^{\mathrm{h}}\right) / 2$, and $E_{\mathbf{k}}^{ \pm}=E_{\mathbf{k}} \pm \delta \xi_{\mathbf{k}}$ with $\delta \xi_{\mathbf{k}}=\left(\xi_{\mathbf{k}}^{\mathrm{e}}-\xi_{\mathbf{k}}^{\mathrm{h}}\right) / 2$. In addition, $v_{\mathbf{k}}^{2}=1-u_{\mathbf{k}}^{2}=\left(1-\xi_{\mathbf{k}} / E_{\mathbf{k}}\right) / 2$. Throughout, we express lengths in units of the effective Bohr radius $a_{0}^{*}$, and energies in units of the effective Rydberg Ry* 24]. In two dimensions the average interparticle spacing is $r_{s}=\left[\pi\left(n_{\mathrm{e}}+n_{\mathrm{h}}\right) / 2\right]^{-1 / 2}$.

Figure 1(a) shows the wave-vector dependence of $\Delta_{\mathbf{k}}$ for equal densities, layer separation $d=1$, and several values of $r_{s}$. These values of $r_{s}$ and a layer sepa-

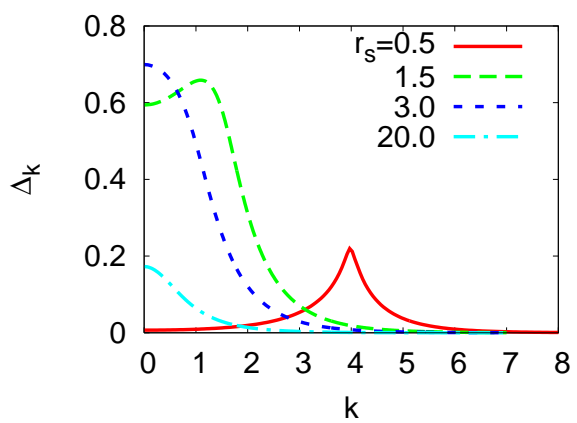

(a)

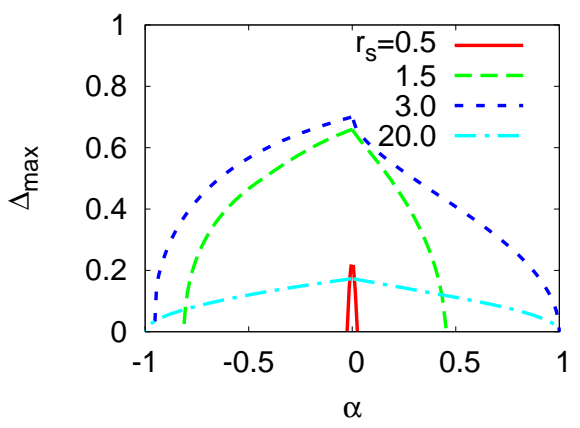

(b)

FIG. 1: (a) Wave-vector dependence of the gap function for $\alpha=0, d=1$, and several values of $r_{s}$; (b) Maximum value $\Delta_{\max }=\max \left\{\Delta_{\mathrm{k}}\right\}$ vs $\alpha$ for $d=1$ and several values of $r_{s}$.

ration of $d=1$ are already experimentally attainable. The wave-vector position $k_{\max }$ of the peak in the gap function is seen to evolve from a finite value in the BCS regime (small $r_{s}$ ) toward zero in the BEC regime (large $r_{s}$ ), while the corresponding value $\Delta_{\max }$ of the gap function attains its maximum for intermediate values of $r_{s}$. This is a generic feature of the density-induced BCS-BEC crossover [25].

We have verified for several values of the distance $1 \leq d \leq 4$ that the optimal value of $r_{s}$ at which $\Delta_{\max }$ attains its largest value is located close to the value of $r_{s}$ where the average chemical potential $\mu=\left(\mu_{\mathrm{e}}+\mu_{\mathrm{h}}\right) / 2$ crosses zero. For $d=1$ this occurs near $r_{s}=3$. This finding is in line with results for the BCS-BEC crossover with a contact potential in three dimensions, namely, that superfluid properties are more robust in the region located between the Fano-Feshbach resonance and the vanishing of the chemical potential [26]. The narrow region lying between the optimal value of $r_{s}$ and the value corresponding to $\mu=0$ thus identifies the middle of the crossover region between the BCS and BEC regimes for the electron-hole bilayer.

The effect of the density imbalance $\alpha \equiv\left(n_{\mathrm{e}}-n_{\mathrm{h}}\right) /\left(n_{\mathrm{e}}+\right.$ $n_{\mathrm{h}}$ ) on $\Delta_{\max }$ is shown in Fig. 1(b). We see that the density imbalance acts to reduce the magnitude of the energy gap and that it has different effects on the two sides of the crossover. In the BCS regime, the mismatch of the Fermi surfaces for electrons and holes strongly affects the 


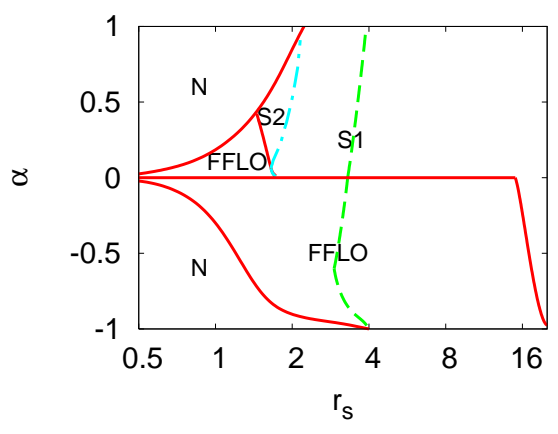

FIG. 2: Zero-temperature phase diagram for $d=1$, showing the stability domains of the different phases: Normal $(\mathrm{N})$, Sarma (S1 and S2), and FFLO. The dashed line corresponds to the curve $\mu=0$, and the dash-dotted line separates the S1 and S2 phase. Note the logarithmic scale for $r_{s}$.

superfluid properties of the system. The superfluid properties are lost when the Fermi energies mismatch becomes large compared with the value of $\Delta_{\max }$ for equal densities. For $r_{s}$ small this occurs for a small value of $\alpha$. In the BEC regime, the superfluid properties are less sensitive to density imbalance, whose main effect is then to reduce the number of electron-hole pairs.

Figure 2 shows the zero-temperature phase diagram for $d=1$. We can identify various phases using $\Delta_{\mathbf{k}}$, determined from Eq. (2), and the superfluid (mass) density $\rho_{s}$. Within mean-field theory and in the zero-temperature limit, $\rho_{s}$ is given by:

$$
\rho_{s}=m_{\mathrm{e}} n_{\mathrm{e}}+m_{\mathrm{h}} n_{\mathrm{h}}-\frac{1}{4 \pi} \sum_{j, \lambda} \frac{\left(k_{j}^{\lambda}\right)^{3}}{\left|\frac{d E_{k}^{\lambda}}{d k}\right|_{k=k_{j}^{\lambda}}} .
$$

Here, $k_{j}^{\lambda}$ is the $j$-th zero of $E_{k}^{\lambda}=0$ with $\lambda=(+,-)$. [For positive (negative) density imbalance only $E_{k}^{+}\left(E_{k}^{-}\right)$has zeros, while no zero occurs for $\alpha=0$.]

The normal phase $(\mathrm{N})$ corresponds to the trivial solution $\Delta_{\mathbf{k}}=0$. The Sarma phases corresponds to nonvanishing $\Delta_{\mathbf{k}}$ when $\alpha \neq 0$ and positive superfluid density $\rho_{s}$. The S1 and S2 denote the Sarma phases for one and two Fermi surfaces, respectively. There will be one zero of $E_{k}^{\lambda}(j=1)$ for the S1 phase (one Fermi surface), and two zeros $(j=1,2)$ for the S2 phase (two Fermi surfaces). Sometimes, the Sarma S2 phase is called the "breached-pair" phase after Ref. [19]. A negative value of $\rho_{s}$ in Eq. (5) indicates that the Sarma phase is unstable toward a phase with a spontaneously generated superfluid current, which we associate [27, 28] with the FFLO phase. We have verified that the Sarma phase, whenever it exists, is always lower in energy with respect to the normal phase. (We recall in this respect that our calculation is at fixed density imbalance, while the original Sarma calculation was at fixed chemical potentials.)
The most prominent feature of Fig. 2 is the marked dependence of the phase diagram on the sign of $\alpha$ [29]. In particular, while the boundary of the normal phase does not depend appreciably on the sign of $\alpha$, the region of stability of the Sarma phase with respect to the FFLO phase depends dramatically on the sign. For $\alpha<0$, the phase diagram is dominated by the FFLO phase, with the Sarma (S1) phase being confined to the extreme BEC region, while for $\alpha>0$, the FFLO phase is compressed into the region of small $r_{s}$.

Such an asymmetry can be understood in terms of the relevant dispersion $E_{\mathbf{k}}^{+}\left(E_{\mathbf{k}}^{-}\right)$for positive (negative) $\alpha$. Due to the mass difference, the term $2 \delta \xi_{\mathbf{k}}$ which is added (subtracted) to $E_{k}$ makes the dispersion $E_{\mathbf{k}}^{+}\left(E_{\mathbf{k}}^{-}\right)$steeper (flatter) with respect to the case of equal masses. As it is clear from the last term in Eq. (5), a flatter dispersion will make the superfluid density more negative, thus replacing the Sarma phase with the FFLO phase. The opposite occurs for positive $\alpha$, when the relevant dispersion becomes steeper.

In this part of the phase diagram there is some room even for the S2 Sarma phase. So far, this phase was found in the literature to be very fragile, being invariably replaced by phase separation or by the development of a FFLO phase [19, 27, 30]. Here, the concurrence of several favorable factors, the mass difference, the wave-vector dependence of the gap, and the intralayer Coulomb repulsion, all serve to stabilize the S2 Sarma phase in an appreciable region of the phase diagram.

We note that the largest number of phases occurs for intermediate values of $r_{s}$ (say, $r_{s}=1.5-3$ ), corresponding to the smooth crossover region between the BCS and BEC limit for $\alpha=0$. For non-zero $\alpha$, several transition lines can be crossed in this region by varying $\alpha$ or $r_{s}$. This is thus the most fertile region to be explored experimentally, also because we recall in the same region $\Delta_{\max }$ is largest and the superfluidity most robust.

In this respect, the behavior of the separate chemical potentials $\mu_{\mathrm{e}}$ and $\mu_{\mathrm{h}}$ vs $\alpha$ can serve to reveal the appearance of a finite value for the gap $\Delta$. This is because $\mu_{\mathrm{e}}$ and $\mu_{\mathrm{h}}$ must show a jump across $\alpha=0$ in order to sustain a finite density imbalance in the superfluid phase. This behavior is shown in Fig. 3 for $d=1$ and $r_{s}=3$, when $\mu_{\mathrm{e}}\left(\alpha=0^{+}\right)-\mu_{\mathrm{e}}\left(\alpha=0^{-}\right) \simeq 2 \Delta_{\max }$. The possible instability toward the FFLO phase we discussed above should not affect the occurrence of this jump. Physical quantities obtained for the Sarma and the FFLO phases should, in fact, merge continuously when $\alpha$ approaches zero, owing to the corresponding vanishing of the modulation wave vector associated with the FFLO phase [28].

It is useful to discuss the sensitivity of our results to the parameters used in the calculations. Increasing the distance $d$ will shift the location of the intermediate region where most phases are seen, to larger values of $r_{s}$ (for $d=2$, e.g., the shift is about a $30 \%$ ), but it does not alter the shape of the phase diagram. Reducing the mass 


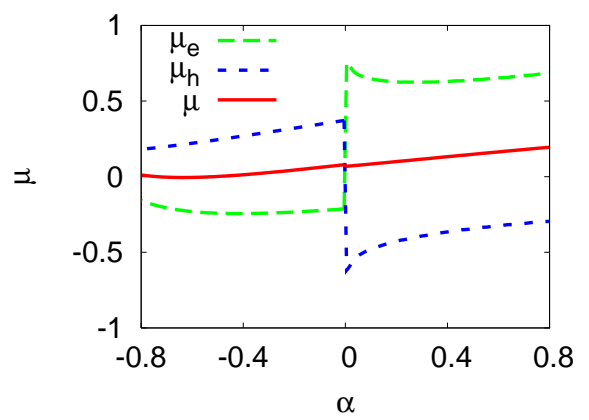

FIG. 3: Zero-temperature electron $\left(\mu_{\mathrm{e}}\right)$ and hole $\left(\mu_{\mathrm{h}}\right)$ chemical potentials vs $\alpha$ for $d=1$ and $r_{s}=3$. The average chemical potential $\mu$ is also shown.

difference has the effect of contracting the FFLO phase for negative $\alpha$ and expanding it for positive $\alpha$. This is as expected from the arguments discussed above. However, even for equal masses the phase diagram changes only quantitatively, allowing even in this case some space for the S2 Sarma phase. Finite temperature assists in stabilizing the Sarma phases with respect to the FFLO: the superfluid density is, in fact, quite sensitive to temperature in the presence of density imbalance. We have found, however, that temperatures below $1 \mathrm{~K}$ do not appreciably alter the phase diagram in the most interesting intermediate $r_{s}$ region. Finally, a reduction of the FFLO phase due to the intra-layer Coulomb repulsion may occur but this reduction should be limited to the region of large $r_{s}$, where the spatial modulation of the gap parameter is expected to be accompanied by a density modulation.

In conclusion, we have shown that electron-hole bilayers are promising candidates for revealing a variety of superfluid phases while traversing the BCS-BEC crossover. Exotic phases such as the FFLO and the S2 Sarma phases which have been so far elusive to experimental detection, should be notably robust in electron-hole bilayer systems.

This work was partially supported by the Italian MIUR with contract Cofin-2005 "Ultracold Fermi Gases and Optical Lattices".

[1] M. Bartenstein et al., Phys. Rev. Lett. 92, 120401 (2004); C.A. Regal, M. Greiner, and D.S. Jin, Phys. Rev. Lett. 92, 040403 (2004); M.W. Zwierlein et al., Phys. Rev. Lett. 92120403 (2004).

[2] L.V. Keldysh and Y.V. Kopaev, Sov. Phys. Solid State 6, 2219 (1965); L.V. Keldysh and A.N. Kozlov, Sov. Phys. JETP 27, 521 (1968).

[3] C. Comte and P. Nozières, J. Phys. (Paris) 43, 1069 (1982); P. Nozières and C. Comte, J. Phys. (Paris) 43, 1083 (1982).

[4] S.I. Shevchenko, Sov. J. Low. Temp. Phys. 2, 251 (1976).

[5] Y.E. Lozovik and V.I. Yudson, JETP Lett. 22, 274
(1975), and Sov. Phys. JETP 44, 389 (1976).

[6] J. Szymański, L. Świerkowski, and D. Neilson, Phys. Rev. B 50, 11002 (1994).

[7] X. Zhu et al., Phys. Rev. Lett. 74, 1633 (1995).

[8] Z.W. Gortel and L. Świerkowski, Surf. Science 361/362, 146 (1996).

[9] S. De Palo, F. Rapisarda, and G. Senatore, Phys. Rev. Lett. 88, 206401 (2002).

[10] L.V. Butov, A.C. Gossard, and D.S. Chemia, Nature 418, 751 (2002); A. T. Hammack et al., Phys. Rev. Lett. 96, 227402 (2006); S. Yiang, et al., cond-mat/0606683

[11] M. Kellogg et al., Phys. Rev. Lett. 93, 036801 (2004); E. Tutuc, M. Shayegan, and D.A. Huse, Phys. Rev. Lett. 93, 036802 (2004); J.P. Eisenstein, Science 305, 950 (2004).

[12] For a review, see J.P. Eisenstein and A.H. MacDonald, Nature 432, 691 (2004).

[13] M. Pohlt et al., Applied Phys. Lett. 80, 2105 (2002).

[14] G. Sarma, J. Phys. Chem. Solids 24, 1029 (1963).

[15] U. Lombardo et al., Phys. Rev. C 64, 064314 (2001).

[16] M.W. Zwierlein et al., Science 311, 492 (2006); G.B. Partridge et al., Science 311, 503 (2006); Y. Shin et al., Phys. Rev. Lett. 97, 030401 (2006).

[17] J. Carlson and S. Reddy, Phys. Rev. Lett. 95, 060401 (2005); C.-H. Pao, Shin-Tza Wu, and S.-K. Yip, Phys. Rev. B 73, 132506 (2006); D. E. Sheehy and L. Radzihovsky, Phys. Rev. Lett. 96, 060401 (2006).

[18] P. Pieri and G. C. Strinati, Phys. Rev. Lett. 96, 150404 (2006); J. Kinnunen, L.M. Jensen, and P. Törmä, Phys. Rev. Lett. 96, 110403 (2006); W. Yi and L. M. Duan, Phys. Rev. A 73, 031604(R) (2006); F. Chevy, Phys. Rev. Lett. 96, 130401 (2006); T. N. De Silva and E. J. Mueller, Phys. Rev. A 73, 051602(R) (2006); M. Haque and H. T. C. Stoof, Phys. Rev. A 74, 011602(R) (2006); J.-P. Martikainen, Phys. Rev. A 74, 013602 (2006); C.-C. Chien et al., Phys. Rev. A 74, 021602(R) (2006).

[19] M. McNeil Forbes et al., Phys. Rev. Lett. 94, 017001 (2005).

[20] D. T. Son and M. A. Stephanov, Phys. Rev. A 74, 013614 (2006); A. Bulgac, M. McNeil Forbes, and A. Schwenk, Phys. Rev. Lett. 97, 020402 (2006); M. Mannarelli, G. Nardulli, and M. Ruggieri, Phys. Rev. A 74, 033606 (2006).

[21] L.V. Butov et al., JETP 92, 260 (2001).

[22] P. Fulde and R.A. Ferrell, Phys. Rev. 135, A550 (1964); A.I. Larkin and Yu.N. Ovchinnikov, Zh. Eksp. Teor. Fiz. 47, 1136 (1964) [Sov. Phys. JETP 20, 762 (1965)].

[23] P. Nozières and F. Pistolesi, Eur. Phys. J. B 10, 649 (1999).

[24] For GaAs-AlGaAs bilayers, $a_{0}^{*}=12.03 \mathrm{~nm}$ and $\mathrm{Ry}^{*}=$ $4.64 \mathrm{meV}$ corresponding to the values $\varepsilon=12.9$ (times the vacuum dielectric constant $\left.\varepsilon_{0}\right), m_{\mathrm{e}}=0.07$ and $m_{\mathrm{h}}=0.30$ (in units of the electron mass).

[25] N. Andrenacci et al., Phys. Rev. B 60, 12410 (1999).

[26] N. Andrenacci, P. Pieri, and G.C. Strinati, Phys. Rev. B 68, 144507 (2003).

[27] S.T. Wu and S. Yip, Phys. Rev. A 67, 053603 (2003).

[28] L. He, M. Jin, and P. Zhuang, Phys. Rev. B 73, 214527 (2006), and references therein.

[29] A similar behavior was recently found for ultracold Fermi atoms by M. Iskin and C.A.R. Sá de Melo, Phys. Rev. Lett. 97, 100404 (2006), and cond-mat/0606624

[30] P.F. Bedaque, H. Caldas, and G. Rupak, Phys. Rev. Lett. 91, 247002 (2003). 